\title{
Incidence, risk factors, and outcomes of jejunal varices of the afferent loop after pancreatoduodenectomy
}

\author{
Mizelle D'SILVA, Yoo-Seok YOON*, Ho-Seong HAN, Jun Suh LEE, Jai Young CHO, Hye Won LEE, Boram LEE, Moon Whan KIM \\ Depatment of Surgery, Seoul National University Bundang Hospital, Seongnam, Korea
}

Introduction: Jejunal varices is a worrisome late complication after pancreaticoduodenectomy (PD) due to recurrent and intractable bleeding. This study was aimed to determine the incidence, risk factors, and outcomes of jejunal varices after PD.

Methods: A total of 709 patients who underwent PD from 2007 to 2017 were enrolled in the study. Preoperative and postoperative follow-up CT images were reviewed to evaluate the development of portal vein (PV) stenosis (> 50\%) and jejunal varices, which was defined as engorged dilated veins $>3 \mathrm{~mm}$.

Results: Jejunal varices developed in 83 (11.7\%) patients after a median duration of 12 (5-21) months after PD. Among those patients, 18 (21.6\%) experienced variceal bleeding. PV stenosis ( $p<0.001$; OR 33.234, 95\% CI 15.568-66.666) and PV/SMV resection $(p=0.028$; OR 2.261, 95\% CI 1.090-4.687) were identified as independent predicting factors for jejunal varices. None of 9 patients who underwent stent placement for PV stenosis before the formation of jejunal varices experienced variceal bleeding, whereas 18 (27.3\%) of 135 patients who did not receive PV stent placement experienced at least one episode of variceal bleeding. Among 17 patients with PV stent placement, variceal bleeding occurred in 3 of 7 patients with stent thrombosis, but not in 10 patients without stent thrombosis.

Conclusions: The results revealed that the incidence of jejunal varices was not low after PD and PV stenosis was a strong risk factor for jejunal varices. Early placement of PV stent and maintenance of stent patency could reduce the risk of variceal bleeding in patients with PV stenosis. 